\title{
Physiological effects of oral glucosamine on joint health: current status and consensus on future research priorities
}

\author{
Yves Henrotin ${ }^{1,2^{*}}$, Xavier Chevalier ${ }^{3}$, Gabriel Herrero-Beaumont ${ }^{4}$, Timothy McAlindon ${ }^{5}$, Ali Mobasheri ${ }^{6}$, \\ Karel Pavelka ${ }^{7}$, Christiane Schön ${ }^{8}$, Harrie Weinans ${ }^{9}$, Hans Biesalski $^{10}$ and Participants at the Hohenheim Consensus \\ Conference in August 29th 2011
}

\begin{abstract}
The aim of this paper was to provide an overview of the current knowledge and understanding of the potential beneficial physiological effects of glucosamine $(G l c N)$ on joint health. The objective was to reach a consensus on four critical questions and to provide recommendations for future research priorities. To this end, nine scientists from Europe and the United States were selected according to their expertise in this particular field and were invited to participate in the Hohenheim conference held in August 2011. Each expert was asked to address a question that had previously been posed by the chairman of the conference. Based on a systematic review of the literature and the collection of recent data, the experts documented the effects of GlcN on cartilage ageing, metabolic/kinetic and maintenance of joint health as well as reduction of risk of OA development. After extensive debate and discussion the expert panel addressed each question and a general consensus statement was developed, agreeing on the current state-of-the-art and future areas for basic and clinical studies. This paper summarizes the available evidence for beneficial effects of GlcN on joint health and proposes new insight into the design of future clinical trials aimed at identifying beneficial physiological effect of GlcN on joint tissues.
\end{abstract}

Keywords: Glucosamine, Osteoarthritis, Treatment, Prevention

\section{Discussion}

Glucosamine (GlcN) may be a suitable intervention for osteoarthritis (OA) and, indeed, is already selfadministered by millions of individuals without any apparent deleterious side-effects. GlcN is and ubiquitous amino sugar that is believed to play a key role in cartilage formation and repair. It is a naturally occurring substance in the body and is essential for the normal growth and repair of connective tissues such as articular cartilage in synovial joints. GlcN is one of the major constituents of the extracellular matrix (ECM) of cartilage and a building block for proteoglycan synthesis. It exists in several forms, GlcN sulfate (GlcN.S) and GlcN hydrochloride (GlcN.H) being the most widely used and

\footnotetext{
* Correspondence: yhenrotin@ulg.ac.be

'Bone and Cartilage Research Unit, University of Liège, Institute of Pathology, Level +5, CHU Sart-Tilman, 4000, Liège, Belgium

${ }^{2}$ Princess Paola Hospital, Marche-en-Famenne, Belgium

Full list of author information is available at the end of the article
}

studied. GlcN.S has been reported to be efficient on knee OA symptoms [1-5].

In this paper, our goal was to explore new avenues concerning the use of GlcN, especially in terms of beneficial and disease preventing physiological effects, notably with respect to OA development. To address this aim, eight experts from six European countries and one expert from the United States were selected and invited according to their expertise in the context of this particular field, to the Hohenheim conference, which was held in August 2011. Each expert was asked to address a question that has been previously posed by the chairman of the conference. Responses were based on review of the published literature and the collection of the most relevant papers and recent relevant data. Following extensive debate and discussion, a general consensus was reached in relation to each question and new strategies were proposed and discussed for basic and clinical future works (Tables 1 and 2).

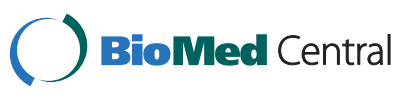




\section{Table 1 Summary of the experts group consensus}

1 At the present time there is no evidence that glucosamine modulates ageing phenotype and no studies have been performed on the effects of glucosamine on aged cartilage.

2 New and more focused studies are clearly needed to determine if GlcN can affect oxidative stress, mitochondrial function, autophagy and responsiveness to cytokines and growth factors.

3 Chondrocytes have the capacity to biosynthesize glucosamine from glucose in health and disease. There is no evidence that chondrocyte glucosamine requirement is modified with age or pathological situation.

4 The endogenous level of glucosamine is comprised between $1 \mu \mathrm{M}$ and $2 \mu \mathrm{M}$, and reach to $10 \mu \mathrm{M}$ after oral administration of a therapeutic dose (1500 mg);

5 There is no information about the optimal dose use and no information of the interference of diet, age and other gastrointestinal comorbidities or association with other nutraceuticals most particularly with chondroitin sulfate on the GlcN phamarcokinetic profile.

6 Glucosamine might contribute to the maintenance of healthy joints, but this should be confirmed in clinical trials designed to investigate its effects on healthy subjects with high risk of osteoarthritis and using parameters, which establish the link between consumption of glucosamine and maintenance of joints (e.g. biochemical markers and/or MRI)

7 In vitro and ex vivo studies on normal and OA chondrocytes demonstrated stimulating effect of GlcN with supra-pharmacologica concentrations on the synthesis of cartilage matrix components and inhibiting potencies on pro-catabolic and pro-inflammatory factors.

8 Prophylactic evidence has been shown in some animal models, but not in human.

9 More research is necessary to explore possible beneficial effects of GlcN in healthy subjects or on risk factors of OA.

This paper addresses some questions related to the evidence on the beneficial effects of GlcN on the joint health. Based on a research of the literature performed on PubMed, the experts attempted to document the effects of GlcN on cartilage ageing and maintenance of joint health, two key points for the substantiation of health claims. They have also provided some suggestions for the design of future clinical trials in order to identify beneficial physiological effects of GlcN on joint tissues.

\section{What is the main impact of cartilage ageing? is GlcN effective against cartilage ageing?}

Ageing is a major contributor to musculoskeletal impairment, the degeneration of joint tissues and the development of OA [6,7]. Age-related changes in articular cartilage contribute to the development and progression of OA. Although the degeneration of articular cartilage is not simply the result of ageing and mechanical wear, it nevertheless modifies the articular joint tissues and synovial fluid $[6,8]$. Although older age is the greatest risk factor for $\mathrm{OA}, \mathrm{OA}$ is not an inevitable consequence of growing old [9]. The mechanisms for the link between ageing and $\mathrm{OA}$ are incompletely understood. Cell stress and oxidative damage contribute to chronic inflammation that promotes age-related diseases. Ageing chondrocytes express a so-called "senescent" secretory phenotype, which has some common characteristics with chondrocyte phenotype in OA, especially in relation to the production and secretion of pro-inflammatory cytokines, chemokines, and proteases [10].

One may question if the oral intake of GlcN could interfere with chondrocyte senescence mechanisms. At this time, it remains difficult to answer the question "is GlcN effective against cartilage ageing or is this compound effective on the ageing phenotype". Indeed there is no published evidence to suggest that GlcN is effective against cartilage or any other connective tissues ageing. Although there is some evidence to suggest that glucose can accelerate in vitro cell senescence, there is no evidence that GLcN can act in one way or another on it. However, considerable evidence for indirect effects of GlcN on ageing has been obtained. A recent proteomic study [11] has shown that GlcN.S alters the expression of proteins involved in signal transduction pathways, redox and stress responses, and protein synthesis and folding processes in human chondrocytes. Interestingly, GlcN affects mainly energy production and metabolic pathways. However, there is no "mechanistic" data linking GlcN.S to the reduction of oxidative stress in in vitro or animal studies.

Another study suggests that alpha-tocopherol, ascorbic acid, selenium, combined GlcN.S and chondroitin exert antioxidant effects on cultured chondrocytes [12]. However, this study used combination products. It is therefore difficult to determine whether GLcN by itself has any direct and specific effects on oxidative stress.

Finally, a study investigated the effects of GlcN.S on heme oxygenase (HO-1), p22 (Phox) (a subunit of NADPH complex) and inducible nitric oxide synthase (iNOS) expression by primary human chondrocytes in vitro [13]. HO-1 gene expression was up-regulated by $10 \mathrm{mmol} / \mathrm{l}$ GlcN.S (24 h). Expression of the p22 (Phox) gene was down-regulated by $10 \mathrm{mmol} / \mathrm{l}$ GlcN.S (48 h treatment); the HO-1 gene was down-regulated by IL- $1 \beta$ and $10 \mathrm{mmol} / \mathrm{l}$ GlcN.S appeared to restore the expression levels to baseline values.

It has recently been described that OA was associated with an alteration in the $\mathrm{O}-\mathrm{N}$-actetyl-glycosaminylation (O-GlcNAc) of proteins in the cartilage of patients [14]. Since the discovery of this protein modification by Torres and Hart [15], O-GlcNAc signalling has been implicated in a diverse array of physiological and pathological functions [16]. Furthermore, there is a growing recognition that global protein O-GlcNAc alterations are involved in the pathophysiology of chronic- and agerelated diseases, such as diabetes, cardiac hypertrophy and failure, immunological disorders, cardiovascular disease, neurodegeneration and cancer $[17,18]$. In this 
Table 2 Consensus and research recommendations for each question addressed Question 1. What is the main impact of cartilage ageing? Is GlcN effective against cartilage ageing? Consensus

Although older age is the greatest risk factor for $\mathrm{OA}, \mathrm{OA}$ is not an inevitable consequence of growing old.

1. Chondrocytes have the capacity to biosynthesize GlcN from glucose in health and disease. There is no evidence that chondrocyte GlcN requirement is modified with age or pathological situation.

2. To study the influence of ageing on the synthesis of GlcN by chondrocytes in in vitro and spontaneous models of OA in animal is recommended.

3. Study of the production of GlcN by chondrocytes at different stages of OA development in animal models should be performed.

4. The senescence-associated secretory phenotype of articular chondrocytes should be better characterized to differentiate ageing and $\mathrm{OA}$ affected cartilage.

5. At the present time there is no evidence that GlcN modulates this phenotype and no studies have been performed on the effects of GlcN on aged cartilage. New and more focused studies are clearly needed to determine if GlcN can affect oxidative stress, mitochondrial function, autophagy and responsiveness to cytokines and growth factors.

6. There is some in vitro evidence that GlcN modulate glucose metabolism is chondrocytes.

7. There is some evidence on systemic effect of GlcN through reduction of inflammatory marker and anabolic effects on cartilage.

\section{Research recommendations}

To investigate GlcN on the following parameters:

- Telomere erosion

- Mitochondrial dysfunction

- Reactive oxygen species (ROS) and antioxidants production

- Responsiveness to anabolic growth factors

- Autophagy

- Apoptosis

- The expression of transcription factors associated with longevity (i.e. SirT1 and FoxO)

- The accumulation Advanced Glycation End products in ECM.

\section{Question 2. How does the metabolic kinetic look like? What about the Cmax levels?}

\section{Consensus}

1. Heterogeneous level of endogenous level of GlcN is comprised between $1 \mu \mathrm{M}$ and $2 \mu \mathrm{M}$

2. Translation of animal models concerning the basal level must been done with caution because there are species differences in GlcN in absorption and bioavailability.

3. There is no information on the interference of diet, age and other gastrointestinal comorbidities on the GlcN phamarcokinetic profile.

Research recommendations

- To study the influence of various diets enriched in GlCN and ageing on the endogenous level of GlcN

- To better quantify the resting plasma levels of GlcN with future developments in MS-technologies and bioanalytical techniques that should contribute to the standardization of assays used to detect.

- To study higher doses of GlcN in human OA to look for beneficial mechanisms of action of GICN.S in experimental models

- To perform more pharmacokinetic and pharmacodynamic studies in human as well as in animals

- To consider the formulation and complexation of the preparations in future in vitro studies on GlcN

- To take into account the serum and synovium levels from clinical trials (physiological effects found around serum levels of $10 \mu \mathrm{M}$ ) to determine the in vitro concentration used in future studies should take into account

- To better investigate the distribution of GlcN in the different joint tissues

Question 3. How can GlcN contribute to cartilage maintenance in healthy subjects, and how can it be demonstrated?

Consensus
patients, but this should be demonstrated in clinical trials designed to investigate its effect on healthy subjects with high risk of $O A$ and evaluating parameters which can establish the link between consumption of GlCN and the maintenance of joints (e.g. biochemical markers and/or $\mathrm{MRI})$. This statement is based on the following observations: Research recommendations

- To investigate the effect of GlcN in the maintenance of healthy subjects. This should be done as recommended in the framework of OARSI, in young subjects with high risk factors of $\mathrm{OA}$

- To investigate the effects of GlcN on a panel of functional imaging and biochemical markers investigating joint tissue metabolism.

- In vitro and ex vivo studies on normal and OA chondrocytes demonstrated stimulating effect of GlcN with supra-pharmacological concentrations on the synthesis of cartilage matrix components and inhibiting potencies on pro-catabolic and pro-inflammatory factors.

- Prophylactic evidence has been shown in some animal models.

- Up-to-date, there is no convincing data demonstrating the potential benefit of GlcN in healthy subjects. 
Table 2 Consensus and research recommendations for each question addressed (Continued)

\begin{tabular}{|c|c|}
\hline Consensus & Research recommendations \\
\hline $\begin{array}{l}\text { 1. No data on modulating risk factors of OA. } \\
\text { 2. Some animal and cases report in human suggest that chronic use of } \\
\text { GLCN in diabetes or "pre-diabetes" individuals might lead to insulin } \\
\text { resistance. However the level of proofs is not sufficient and we lack long } \\
\text { term studies of GlcN use for individuals with diabetes or pre diabetes. } \\
\text { Based on the available evidence, no specific recommendation for } \\
\text { controlling glycemia in patients taking GlcN could be addressed }\end{array}$ & $\begin{array}{l}\text { - To search the influence of GlcN on metabolic (e.g. adipokine secretions, } \\
\text { systemic inflammation) and structural risk factors (e.g.; osteophytes, bone } \\
\text { marrow lesions) } \\
\text { - To identify surrogate marker for risk factors investigation (e.g. multiplex } \\
\text { biological test including markers of inflammation, glucose, fat tissue, } \\
\text { bone and cartilage metabolism or aggregate score integrating biological, } \\
\text { imaging and clinical) } \\
\text { - To design clinical trials on well-defined subgroups with a specific risk } \\
\text { profile (metabolic syndrome, etc.) to demonstrate beneficial effects of } \\
\text { GlcN on risk factors or risk profile. }\end{array}$ \\
\hline
\end{tabular}

sense, GlcN has been described to increase the flux through the hexosamine pathway modifying the amount of O-GlcNAc acylated proteins, and thus acting as a protective agent against cell stress and promoting cell survival [19]. Preliminary data from Herrero Beaumont's laboratory show that glucosamine treatment could diminish OA damage in parallel with a diminution in O-GlcNAc modified proteins (Largo, unpublished data).

Another indirect effect of GlcN on ageing could happen through its effect on inflammation. A number of recent publications described anti-inflammatory effects of high dose of GlcN in rheumatoid arthritis models [20,21]. These findings are underlined by studies with physiological concentrations of GlcN.S where increased levels of mRNA and aggrecan core protein and a decrease of the production of MMP-3 in human OA chondrocytes were investigated [22]. The study of Byron et al. using equine chondrocytes and synovial cells found that GlcN.H decreased the IL-1-stimulated production of prostaglandins (PGEs) in both cell types [23].

\section{Do GLCN interfere with glucose metabolism in chondrocytes?}

A key question is "is GlcN capable to interfere with glucose metabolism of chondrocytes?". Chondrocytes are highly glycolytic and glucose is an important metabolic fuel for fully differentiated chondrocytes both during postnatal development and in adult articular cartilage [24]. Glucose is also a common structural precursor for the synthesis of extracellular matrix glycosaminoglycans (GAGs) [24]. In addition, chondrocytes express the insulin receptor and are very sensitive to extracellular and intracellular glucose levels [25]. Healthy chondrocytes have a negative feedback loop for controlling the uptake of glucose by modulating the expression of glucose transporter-1 (GLUT-1), depending on extracellular glucose levels. Therefore, if extracellular glucose increases, chondrocytes induce the degradation of GLUT1. However in OA chondrocytes, this feedback appears to be disturbed. Hence, under high glucose, GLUT-1 transporters cannot adequately be down regulated and intracellular glucose accumulation occurs with subsequent deleterious effects [26,27]. This is an extremely interesting phenomenon that might have major repercussions for the aged population where undetected diabetes (i.e. subclinical, mild) and early OA occur frequently together. This fact might explain a potential mechanism of action of GlcN that shares the GLUT transporter system with glucose. Thus high concentrations of GlcN.S and GlcN.H might potentially act as a competitive inhibitor for glucose uptake [28] and protect chondrocytes against high glucose. Intracellular competition may also occur at substrate level since glucose and GlcN are both phosphorylated by the same enzyme, glucokinase (hexokinase). However, in turn, phosphorylated GlcN is an allosteric inhibitor of glucokinase. The consequence of this is a negative feedback loop that blocks glycolysis [29,30]. Nevertheless, different forms of GlcN might act somehow differently since $\mathrm{N}$-acetyl-GlcN is phosphorylated by a different enzyme preventing the intracellular competition with glucose [31]. It might be possible that GlcN can achieve both, activating insulin resistance and/or blocking glycolysis, depending on cell phenotype, disease status and specific form of the amino sugar.

\section{How does the metabolic kinetic look like? What about the Cmax levels?}

GlcN is highly active when added to cell cultures or used in animal models at high concentrations [32]. While several studies have published information about the mechanism of action of GlcN, its pharmacokinetics has only been recently described. The lack of sensitive analytical methods capable of detecting the compound in biological fluids has been the primary limitation of pharmacokinetic studies. In addition, other major difficulties associated with the quantitative determination of GlcN in biological samples are low GlcN levels and the presence of many structurally related sugar molecules that can interfere with its analysis $[33,34]$. 
Persiani et al. described the complete pharmacokinetic profile of GlcN, after three oral doses (once daily, consecutive administration) of $1500 \mathrm{mg}$ crystalline GlcN.S in healthy volunteers [35]. GlcN is bioavailable from orally administered crystalline GlcN.S with maximum plasma concentrations up to 100-fold higher than endogenous levels, in the $10 \mu \mathrm{M}$ range after approximately $3 \mathrm{~h}$ post ingestion. Pharmacokinetic parameters indicate that it is eliminated with a half-life estimated of approximately $15 \mathrm{~h}$, thus supporting the once-daily dosing. Finally, the pharmacokinetics was linear in the dose range of 750-1500 mg of GlcN.S, but not clearly defined at higher doses [35].

Older techniques were not sensitive enough to monitor GlcN plasma concentrations giving erratic values. Previous human and animal studies failed to detect circulating endogenous GlcN.S [35-37] or GlcN.H [38] at concentrations ranging from as low as 10 to as high as $200 \mathrm{ng} / \mathrm{ml}$. The potential pathophysiological significance of this variability should be further investigated. Repeated once-daily doses of around $20 \mathrm{mg} / \mathrm{kg}$ GlcN.S showed a similar pharmacokinetic profiles with a peak plasma concentration (Cmax) around $10 \mu \mathrm{M}$, while the GlcN concentration in synovial fluid was only $23.5 \%$ lower than that in plasma $[39,40]$. Therefore, according to published results, GlcN is well absorbed after repeated oral administration. Furthermore, taking into account other pharmacokinetic studies in humans, the Cmax after a once daily dosing of $20 \mathrm{mg} / \mathrm{kg}$ GlcN.S is about $10 \mu \mathrm{M}$ [32].

The National Institutes of Health (NIH)-sponsored the GlcN hydrochloride (GlcN.H)/Chondroitin sulfate Arthritis Intervention Trial (GAIT), which was aimed at studying the effect of these two compounds on pain and function in knee OA patients. As part of this initiative, pharmacokinetic studies were undertaken. GlcN concentrations found in this study were much lower than those found in patients treated with GlcN.S $1500 \mathrm{mg}$. Thus, after a single-dose of GlcN.H $500 \mathrm{mg}$, the mean plasma Cmax was $3 \mu \mathrm{M}$ in healthy volunteers. Moreover, the Cmax and the area under the curve of GlcN were even lower when the GlcN.H was given chronically in fractioned doses of $500 \mathrm{mg}$ three times daily reaching a Cmax of $211 \mathrm{ng} / \mathrm{mL}$ [41]. The mean plasma Cmax was achieved after a single dosage of $1500 \mathrm{mg}$ GlcN.H, given orally with 6 capsules of $250 \mathrm{mg}$ each after a threemonth treatment. In addition, the bioavailability of GlcN was decreased in patients who took the compound in combination with chondroitin sulfate [41].

Therefore a topical and crucial question is whether 10 $\mu \mathrm{M}$ GlcN has biological effects in in vitro systems, animal models, and in human subjects. Animal studies showing significant effects of GlcN not only at cartilage level in OA models but also in other systems were achieved at doses
15- and 20-fold greater than typical doses consumed by human subjects $[42,43]$. Most in vitro studies of GlcN.S and GlcN.H activity on joint tissue and chondrocytes have been performed in the 500 and $5000 \mu \mathrm{M}$ range [32,44]. Some studies have also described some beneficial effects of GlcN.S and GlcN.H at in vitro concentrations around $50 \mu \mathrm{M}$ and even as low as $10 \mu \mathrm{M}[23,32]$. Nonetheless, the majority of published papers report much higher GlcN doses leading to high concentrations, far from those achieved after the recommended dosing in human OA treatment. For this reason, more studies are required to assess the biological activity of $10 \mu \mathrm{M}$ GlcN in order to gain mechanistic insight of its actions on OA joint tissues [43].

Finally several key questions remain unanswered. Is ageing related to a decrease of endogenous GlcN synthesis? Do changes in eating habits contribute to elevated requirements of exogenous GlcN? Is endogenous GlcN enough to supply chondrocyte metabolism? At the present time these questions remain unanswered. These points should be clarified in future studies to estimate whether administering GlcN is pertinent and whether GlcN provides beneficial effects on joint health.

\section{How can GlcN contribute to cartilage maintenance in healthy subjects and how can this be demonstrated?}

There are currently no studies that have tested GlcN as a chondroprotective agent in a preventive context. Nevertheless, there is some indirect evidence from published trials, showing a possible role of GlcN as a preventive agent in the progression of knee OA. GlcN.S indeed prevented joint space narrowing (JSN) in two randomised, placebo controlled, double blind trials of 3year duration in knee OA $[1,2]$. Several important points can be gleaned from these trials. The first one is the mild to moderate characteristics of the patient population at baseline. The effects observed in this population may therefore be extrapolated, with some caution, to the general population at risk for OA. The second point is that the subgroup analysis of postmenopausal female patients showed more effects on joint structure in this subgroup than in the overall population [45]. Being a female carries a greater risk factor for the development of OA and the effects observed in this subgroup of patients may be predictive of the response to treatment or of its preventive effect if the treatment is initiated earlier. The third point is that some effects have been observed on the contralateral knee in these two long-term trials with GlcN.S. The minimum joint space width (JSW) of the contralateral knee was $4.72 \pm 1.52 \mathrm{~mm}$, which is similar to the values observed in the general population. The effect of GlcN.S on the contralateral knee was not significantly different from placebo, but there was a trend for prevention of OA $(\mathrm{p}=0.17)$. The fourth point is that the 
structure modifying effect of GlcN.S was particularly evident in these patients with better-preserved joint space width at baseline, with joint structure closer to that of the general population [46]. The fifth point is that the patients in these two long-term studies were followed for another 5 years after the termination of the 3-year trials. Pooled incidence of total knee replacement during a mean follow up was lower in GlcN.S-treated population than in placebo group $(14.5 \%$ vs. $6.3 \%$, p $=0.024)$ [47]. However, one important limitation of this kind of study is that in the follow up period the patients have been treated in different ways. The debate about possible clinical efficacy is based on two 3-year studies, but recent analysis of 4 structure-modifying trials did not confirm the preventive effect of GlcN.S or GlcN.H [48].

Recently, the EFSA panel of experts has published its scientific position in relation to the substantiation of a health claim related to GlcN and maintenance of joints [49]. The claimed effect was that GlcN "contributes to the protection of joint cartilage exposed to excessive motion or loading and helps to improve the range of motion in joints". The target population proposed by the applicant was healthy individuals exposed to excessive load on the joint. Maintenance of joints was a beneficial physiological effect. The applicant provided two human studies as pertinent to the claim. The first study was a randomized, double-blind, placebo-controlled trial [50] including 121 male patients who had a recent history of acute knee sport injury. They received either $1.5 \mathrm{~g} \mathrm{GlcN}$ $(n=62)$ per day or placebo (cellulose; $n=59)$ for 28 days. Pain and functional ability were evaluated at the beginning of the study and at 7, 14, 21 and 28 days. Supplementation with GlcN demonstrated significant improvement in knee flexion and extension but not in pain and swelling. The EFSA panel of experts considered that the provided evidence did not establish that patients with acute knee injury are representative of the target population with regards to the status of joint tissues or that results obtained in studies on subjects with acute knee injury can be extrapolated to the proposed target population (healthy individuals exposed to excessive load on the joints). In the second human study [51], 21 male soccer players (19-22 years of age, mean 20.3) and as, a control group, 10 male college students (20-27 years of age, mean 23.5) were recruited. They carried out an open label intervention with GlcN.H. To this end, the subjects were divided into two groups that received $1.5 \mathrm{~g}$ $(n=9)$ or $3 \mathrm{~g}(\mathrm{n}=10) \mathrm{GlcN}$ per day for three months. Urinary samples were taken at baseline, after the GlcN administration (at three months) and three months after withdrawal of GlcN administration (at six months). The end point of the study involved measurement of the urinary concentrations of CTX-II, C2C, two markers of type II collagen degradation, CPII, a marker of type II collagen synthesis, and the ratio CTX-II/CPII. The administration of 1.5 to $3 \mathrm{mg}$ of GlcN significantly decreased CTX-II levels $(\mathrm{p}<0.01)$ [51] but the effect was transient and disappeared after withdrawal of the administration. The panel has noted that this intervention study was not adequately controlled for factors that may have influenced the urinary analysis over the duration of the study, and that the provided evidence did not establish that changes in urinary CTX-II over a period of three months can predict significant changes in type II collagen in joint cartilage. The panel concluded that a cause and effect relationship has not been established between the consumption of GlcN and the maintenance of joint health because of missing appropriate clinical trials in the target (i.e. healthy) population. Besides some evidence for the beneficial effects of $\mathrm{GlcN}$, the remarks of the EFSA outline the limitations of clinical investigations to demonstrate maintenance of joint health.

\section{Can GIcN reduce the risk of OA development?}

The question as to whether GlcN has effectiveness to prevent OA development has not been tested specifically among human subjects. Therefore, it is necessary to consider the processes by which OA develops, and make extrapolations from observations made in animal models and other types of studies about how GlcN might have efficacy in this situation.

With respect to the potential of an intervention as a preventive strategy for $\mathrm{OA}$, it is important to realize that such an effect could be mediated either through an influence on the risk factor profile, or through biological mechanisms that interact directly with pathophysiological processes in OA.

The effects of GlcN on other constitutional characteristics that might represent risk factors for OA (such as body weight) are unlikely or untested, with the possible exception of systemic inflammation. Elevated C-reactive protein levels have been associated with OA [52] and with the progression of hand and wrist OA [53]. The pathophysiological basis of this relationship is uncertain, but it is possible that it reflects the effects of adiposemediated inflammation on articular structures, or, alternatively, some level of synovitis. In addition, studies during the past 2 years have uncovered diverse pathways that cause chondrocytes to become activated and produce pro-inflammatory mediators, through activation of inflammatory genes regulated by transcription factors such as NF-kB [54]. Furthermore, animal studies [55,56], and one human clinical trial [57], have suggested that the suppression of inflammation may reduce structural progression of OA. It has been proposed that $\mathrm{GlcN}$ might be able to intervene in these inflammatory pathways through a number of mechanisms. For example, Imagawa et al. found that O-GlcNAc could prevent 
cytokine-induced demethylation of a specific CpG site in the IL-1 $\beta$ promoter and this was associated with decreased expression of IL-1 $\beta$ [58]. Other investigators have generated in vitro evidence that GlcN.S attenuates NF- $\mathrm{kB}$ activation at concentrations comparable to those seen in patients following oral supplemental ingestion [59].

Due to the competition between GlcN and glucose not only at the level of cellular uptake through the GLUT transporters but also in the hexosamine pathway (one of the alternative routes of glucose metabolism), patients treated with this compound may have increased insulin resistance, as suggested by findings in some animals studies, although not in others $[42,60,61]$. It is therefore important to address the question: "Could oral intake of GlcN interfere with metabolic syndrome and insulin resistance?" With respect to any influence on an individual's risk factor profile, there is a potential for an adverse interaction of GlcN with aspects of the metabolic syndrome, which is common among elderly people with OA [62]. Effects of GlcN on insulin sensitivity through the hexosamine biosynthetic pathway have been reported in animal models [63]. Indeed, intravenous GlcN was used to produce an animal model for insulin resistance and diabetes as part of early research in that field. It is important to investigate the potential negative side effects of GlcN on glucose metabolism, in particular in diabetic and 'pre-diabetic' patients. Several studies have tested this possibility. Studies from Scroggie et al. [64] and Muniyappa et al. [65] showed no effect on insulin resistance after GlcN use, whereas Pham et al. [66] reported data indicating that GlcN worsens insulin sensitivity at doses that are being taken for OA.

In one metabolic study of oral GlcN on serum glucose and insulin levels among 16 patients with OA, three participants demonstrated significant incremental elevations in glucose levels after ingestion of GlcN.S [67]. The authors concluded that GlcN ingestion might affect glucose levels and consequent glucose uptake in patients who have untreated diabetes or glucose intolerance. Dostrovsky et al. recently performed a systematic review to determine if exogenous GlcN adversely affects glucose metabolism in humans [68]. They included 11 studies (6 randomized controlled trials and 5 prospective studies). Four found decreased insulin sensitivity or increased fasting glucose in subjects taking GlcN. They concluded that the available evidence is mixed with regard to the effect of oral GlcN on glucose metabolism, and that more studies are needed, particularly some including subjects at high risk for impairment in glucose homeostasis.

In contrast, high intravenous and intra-arterial doses of GlcN did not affect insulin sensitivity, secretion or action $[69,70]$. Another study in healthy volunteers that received orally repeated once-daily doses of $1500 \mathrm{mg}$ GlcN.S did not show any changes in serum insulin or blood glucose levels [71]. In turn, patients with Type 2 diabetes receiving a combination of GlcN and chondroitin sulfate for 3 months had no change in their diabetes management or hemoglobin A1c concentrations [64]. Finally, fasting plasma glucose levels were not modified in short-term clinical trials with GlcN.S, as well as in the Reginster long-term trial [1]. In contrast, Pavelka reported that four patients developed diabetes during a 3 -year study; however, three of them were in the placebo group and only one was on GlcN.S treatment [2]. A systematic literature review suggests that GlcN has no effect on fasting blood glucose levels, glucose metabolism, or insulin sensitivity at any oral dose level in healthy subjects, individuals with diabetes, or those with impaired glucose tolerance [61]. As concluded in the latter reference, the available evidence implicating GlcN as a diabetogenic agent are limited to rodent infusion studies and in vitro observations, experimental models that were determined not to be relevant to humans. Longitudinal studies in large cohorts of healthy human subjects are therefore needed.

Some animal studies and human case reports suggest that chronic use of GlcN in diabetic or "pre-diabetic" individuals might lead to or exacerbate insulin resistance. However the level of proof is not sufficient and we lack long-term studies of GlcN use in individuals with diabetes or pre-diabetes. Based on the available evidence, no specific recommendation for controlling glycemia in patients taking GlcN is proposed.

The existence of the conflicting published reports highlights the paucity of information and the need for further studies in this area.

\section{Concluding remarks}

GlcN is one of the most widely used "over the counter" dietary supplements for the management of OA. In some European countries GlcN is a registered drug. The aim of the Hohenheim Conference and this consensus paper was not to explore one more time the efficacy of GlcN on symptoms and structure in patients with knee or hip OA. This has already been done numerous times in meta-analyses and literature reviews, including a Cochrane review [72,73]. Despite the extensive studies and interest in this compound, its use is still a matter for debate. The aim of this conference was to provide consensual comments on the potential beneficial effect of GlcN on joint health (Tables 1 and 2).

The first question to address was whether normal chondrocytes need GlcN for their physiological function and metabolism. The answer is clearly no because cells favour glucose as the main fuel for energetic production and production of cartilage proteoglycans [24]. In 
presence of normal glucose concentration, the uptake of GlcN is completely inhibited [74]. Substrate level competition may operate in situations where there is a local decrease in glucose levels or a significant increase in the local concentration of GlcN (millimolar concentrations). Another aspect of the question concerns GlcN and ageing chondrocytes. During the process of chondrocyte senescence, several factors (cell apoptosis, oxidative stress, decreased responsiveness to growth factors, telomere erosion, glycation of ECM macromolecules) contribute to the modification of ECM production in a quantitative and qualitative manner and, as a consequence, this may lead to decreased capacity of the cartilage repair. The lesions observed in ageing cartilage seem similar to those observed in OA cartilage. Thus cartilage ageing is a major risk factor of OA but for many scientists it is still considered to be a different condition to OA. It is therefore important to develop novel tools and assays for mechanistic in vitro studies on the effect of GlcN on cartilage ageing. It will be also interesting to see whether GlcN can influence the production of advance glycation end products (AGEs).

The local anti-inflammatory action of GlcN has clearly been demonstrated by many in vitro studies [44,58,75-78]. An important consideration of future researches could be more in-depth studies of the systemic and general anti-inflammatory effects of GlcN. This will be interesting in the context of a link between metabolic syndrome and knee OA; it will be indeed important to explore whether GlcN might be able to interfere with metabolic syndrome. One proposal could be to observe the systemic effect of GlcN, for instance on ultrasensitive CRP, a marker of molecular inflammation associated with metabolic syndrome. Improving this molecular inflammatory state in patients with knee OA and metabolic syndrome could be an important target of future studies. This potential general effect of GlcN surely needs to be better explored. A recent study showed that high intake of GlcN in rabbits with both articular and vascular disease, is able to diminish the general inflammation and reduce atheromatous plaques, suggesting that oral GlcN.S may exert systemic anti-inflammatory actions [42].

In the line of a potential systemic effect of $\mathrm{GlcN}$, data regarding the extra articular actions of GlcN, especially on muscle are missing. A recent work shows that in patients suffering from knee OA, GlcN.S reduced pain and improved muscle strength with resistance training in a randomized placebo controlled trial [79]. GlcN.S might also act at distance of the joint, in tissues where its concentration is high such as intestine and liver [38]. This might represent an interesting approach in the future.

How and to whom should we propose any preventive effect of GlcN? Our proposal is to stratify and select some populations without symptomatic OA but submitted to a risk factor, which could potentially be influenced by GlcN. Thus, individuals with a history of traumatic joint injuries (i.e. meniscal tears, anterior cruciate ligament injury) or subjects engaged in high load ballistic sports with repetitive joint impact presenting a higher risk of cartilage degradation may be targeted. In this setting, GlcN may present a beneficial effect on joint structure since we hypothesize that oral GlcN is locally available into the joint and may favour cartilage repair. Similarly, individuals with early OA (doubtful stage 1 in the Kellgren-Lawrence classification system) could be a target for preventive treatment with GlcN. Ageing people without overt OA is also a potentially good population to target. Finally, individuals with metabolic syndrome constitute an interesting population to follow, since GlcN may demonstrate not only local but also systemic effects. One key (but difficult) question is to determine which surrogate biomarkers should be adopted to demonstrate the clinical benefits of GlcN on OA development. In other words, how can we detect small variations in cartilage metabolism that could potentially indicate increased cartilage turnover in normal subjects? So far no such biomarkers have been validated in normal subjects. Assays for several biological molecules have been developed as indicators of cartilage and bone metabolism in OA patients [80]. A recent study comparing soccer players (without knee pain and without radiological OA) to healthy but non-sporty young subjects, has shown that treatment with GlcN for 3 months decreases type II collagen degradation (as measured by urinary CTX-II levels) but GlcN did not affect the increase of type II collagen synthesis (reflected by CP-II serum level) [51]. We propose that a combination of biological markers such as Coll2-1, Coll2-1 $\mathrm{NO}_{2}, \mathrm{COMP}$, HA or fibuline-3 fragments (Fib3-1 and Fib3-2) should be used as surrogate biomarkers of cartilage function in such studies $[4,81]$. In addition, sophisticated and new imaging modalities such as MRI with dGemric (delayed gadolinium-enhanced MRI) would be interesting to use in this context for examining the content of biological markers associated with load-bearing (i.e. proteoglycans and their constituent GAGs) in cartilage. The volume of cartilage, the presence and severity of synovitis and/or bone marrow lesions (BML) could also be investigated by MRI. In a recent and relevant study performed in obese patients without symptomatic knee OA (only $32 \%$ present radiographic knee OA), it was demonstrated that weight loss has structure modifying effects as expressed by a gain in the medial dGemric index which indirectly reflects the GAG content of cartilage [82]. This kind of study using a surrogate marker such as dGemric index is typically one that could be applied for studying the structure modifying properties of $\mathrm{GlcN}$ in patients 
without overt OA but those exposed to one or more risk factors for developing OA. The use of scintigraphy with specific binding of macrophages may also be interesting in future studies on GlcN [83]. The goal of this kind of study should be to demonstrate that long-term treatment with GlcN may favourably influence some markers of cartilage metabolism, a prerequisite condition that would be indicative but not conclusive of a chondroprotective effect.

The final question concerns the type of GlcN and the dosage of the molecule that should be recommended. GlcN salts are hydrolysed by the acidic $\mathrm{pH}$ of the gastric cavity and therefore it is probable that both GlcN.H and GlcN.S result in the same form preceding subsequent intestinal absorption and liver metabolism. A recent paper showed bioequivalence of the two forms. Both in rats and in human volunteers, a single dose charge of GlcN.OH or GlcN.S results in the same levels of GlcN excretion in the urine [84]. Therefore, there is not sufficient data to differentiate between the two forms of $\mathrm{GlcN}$, at least from a pharmacokinetic point of view. Absorption of GlcN after oral administration is limited, leading to low bioavailability (varying between animal species and human).

GlcN remains a controversial molecule; general conclusions and perspectives are to move toward new types of clinical trials in healthy subjects that may be able to explore the potential role of GlcN, considered as a nutriment, to prevent the secondary development of osteoarticular diseases. We have proposed in the present paper some statements and proposals for further studies.

\footnotetext{
Abbreviations

AGE: Advance glycation end-product; Cmax: Maximum plasma concentration; ECM: Extracellular matrix; Fib: Fibuline;

GAG: Glycosaminoglycan; GAIT: Glucosamine hydrochloride/chondroitin sulfate Arthritis Intervention Trial; GlcN: Glucosamine; GlcN.H: Glucosamine hydrochloride; GICN.S: Glucosamine sulfate; GLUT: Glucose transporter; $\mathrm{HO}$ : Heme oxygenase; iNOS: Inducible nitric oxide synthase; JSN: Joint Space Narrowing; JSW: Joint Space Width; NIH: National Institutes of Health; OA: Osteoarthritis; O-GlcNAc: O-N-acetylglycoaminylation; PGE: Prostaglandin.
}

\section{Competing interests}

$Y$ Henrotin is the founder of Artialis SA a spin-off of the University of Liège. His research has been supported by educational grant from Bioiberica, Nestle, Royal Canin, Expanscience, Danone and BioXtract. The other authors have no conflict of interest related to the subject of this paper. A. Mobasheri acknowledges the financial support of The Wellcome Trust, the National Centre for the Replacement, Refinement, and Reduction of Animals in Research (NC3Rs; grant number: Mobasheri. A. 28102007), the Biotechnology and Biological Sciences Research Council (BBSRC; grants BBSRC/S/M/2006/ 13141 and BB/G018030/1) and the Engineering and Physical Sciences Research Council (EPSRC). Y Henrotin, H Weinans and A. Mobasheri are members of the D-BOARD Consortium funded by European Commission Framework 7 Programme (EU FP7) and wish to acknowledge funding from the Commission in relation to project number 305815, (Novel Diagnostics and Biomarkers for Early Identification of Chronic Inflammatory Joint Diseases, HEALTH.2012.2.4.5-2).

\section{Authors' contributions}

$\mathrm{YH}$ chaired the consensus conference, formulated the questions and compiled the answers to the questions. HW and AM addressed question 1. GHB addressed question 2. PK has written the answer to question 3. MT wrote the answer to question 4. XC wrote the concluding remarks. SC reviewed the draft and compiled the consensus remarks and research recommendations. $A M$ and $T M$ edited the final version of the manuscript. $H B$ co-chaired the consensus conference. All authors have read and approved the final manuscript.

\section{Acknowledgements}

The authors thank Christelle Boileau for her assistance in preparing the manuscript.

\section{Author details}

'Bone and Cartilage Research Unit, University of Liège, Institute of Pathology, Level +5, CHU Sart-Tilman, 4000, Liège, Belgium. ²Princess Paola Hospital, Marche-en-Famenne, Belgium. ${ }^{3}$ Rheumatology Department, Mondor Hospital, Créteil, France. ${ }^{4}$ Bone and Joint Research Unit, Service of Rheumatology, Fundación Jiménez Díaz, Universidad Autónoma, Madrid, Spain. ${ }^{5}$ Division of Rheumatology, Tufts Medical Center, Box 406800 Washington Street, Boston, MA 02111, USA. ${ }^{6}$ Musculoskeletal Research Group, Division of Veterinary Medicine, School of Veterinary Medicine and Science, Faculty of Medicine and Health Sciences, The University of Nottingham, Sutton Bonington Campus, Sutton Bonington, Leicestershire LE12 5RD, United Kingdom. ' Institute of Rheumatology, Prague, Czech Republic. ${ }^{8}$ UMC Utrecht, Department of Orthopedics, Heidelberglaan 100, 3584 CX, Utrecht, The Netherlands. ' $E$ Erasmus University Medical Center, Rotterdam, The Netherlands. ${ }^{10}$ Department of Biological Chemistry and Nutrition, Garbenstrasse 30, D 70593, Stuttgart, Germany.

Received: 15 October 2012 Accepted: 7 March 2013 Published: 26 March 2013

\section{References}

1. Reginster JY, Deroisy R, Rovati LC, Lee RL, Lejeune E, Bruyere O, Giacovelli G, Henrotin Y, Dacre JE, Gossett C: Long-term effects of glucosamine sulphate on osteoarthritis progression: a randomised, placebo-controlled clinical trial. Lancet 2001, 357:251-256.

2. Pavelka K, Gatterova J, Olejarova M, Machacek S, Giacovelli G, Rovati LC: Glucosamine sulfate use and delay of progression of knee osteoarthritis: a 3-year, randomized, placebo-controlled, double-blind study. Arch Intern Med 2002, 162:2113-2123.

3. Clegg DO, Reda DJ, Harris CL, Klein MA, O'Dell JR, Hooper MM, Bradley JD, Bingham CO 3rd, Weisman MH, Jackson CG, Lane NE, Cush JJ, Moreland LW, Schumacher HR Jr, Oddis CV, Wolfe F, Molitor JA, Yocum DE, Schnitzer TJ, Furst DE, Sawitzke AD, Shi H, Brandt KD, Moskowitz RW, Williams HJ: Glucosamine, chondroitin sulfate, and the two in combination for painful knee osteoarthritis. N Engl J Med 2006, 354:795-808.

4. Henrotin Y, Mobasheri A, Marty M: Is There Any Scientific Evidence For the Use of Glucosamine in the Management of Human Osteoarthritis. Arthritis Res Ther 2012. In press.

5. Altman RD, Abramson S, Bruyere O, Clegg D, Herrero-Beaumont G, Maheu E, Moskowitz R, Pavelka K, Reginster JY: Commentary: osteoarthritis of the knee and glucosamine. Osteoarthr Cartil 2006, 14:963-966.

6. Hamerman D: Biology of the aging joint. Clin Geriatr Med 1998, 14:417-433.

7. Lotz MK, Carames B: Autophagy and cartilage homeostasis mechanisms in joint health, aging and OA. Nat Rev Rheumatol 2011, 7:579-587.

8. Buckwalter JA, Mankin HJ: Articular cartilage: degeneration and osteoarthritis, repair, regeneration, and transplantation. Instr Course Lect 1998, 47:487-504.

9. Shane Anderson A, Loeser RF: Why is osteoarthritis an age-related disease? Best Pract Res Clin Rheumatol 2010, 24:15-26.

10. Loeser RF: Aging and osteoarthritis. Curr Opin Rheumatol 2011, 23:492-496

11. Calamia V, Ruiz-Romero C, Rocha B, Fernandez-Puente P, Mateos J, Montell E, Verges J, Blanco FJ: Pharmacoproteomic study of the effects of chondroitin and glucosamine sulfate on human articular chondrocytes. Arthritis Res Ther 2010, 12:R138.

12. Graeser AC, Giller K, Wiegand H, Barella L, Rimbach G, Boesch Saadatmandi $\mathrm{C}$ : Synergistic chondroprotective effect of alpha-tocopherol, ascorbic acid, and selenium as well as glucosamine and chondroitin on oxidant 
induced cell death and inhibition of matrix metalloproteinase-3-studies in cultured chondrocyte. Molecules 2010, 15:27-39.

13. Valvason C, Musacchio E, Pozzuoli A, Ramonda R, Aldegheri R, Punzi L: Influence of glucosamine sulphate on oxidative stress in human osteoarthritic chondrocytes: effects on HO-1, p22(Phox) and iNOS expression. Rheumatology (Oxford) 2008, 47:31-35.

14. Tardio L, Herrero-Beaumont G, Gómez-Barrena E, Andrés-Bergós J, Largo R: Osteoarthritis leads to increased levels of protein O-linked Nacetylglucosamine in the cartilage. Osteoarthr Cartil 2010, 18:S111.

15. Torres CR, Hart GW: Topography and polypeptide distribution of terminal $\mathrm{N}$-acetylglucosamine residues on the surfaces of intact lymphocytes. Evidence for O-linked GlcNAc. J Biol Chem 1984, 259:3308-3317.

16. Hart GW, Housley MP, Slawson C: Cycling of O-linked beta-N -acetylglucosamine on nucleocytoplasmic proteins. Nature 2007, 446:1017-1022.

17. Hanover JA, Krause MW, Love DC: The hexosamine signaling pathway: OGlcNAc cycling in feast or famine. Biochim Biophys Acta 2010, 1800:80-95.

18. Zachara NE, Hart GW: Cell signaling, the essential role of O-GIcNAc. Biochim Biophys Acta 2006, 1761:599-617.

19. Chatham JC, Not LG, Fulop N, Marchase RB: Hexosamine biosynthesis and protein O-glycosylation: the first line of defense against stress, ischemia and trauma. Shock 2008, 29:431-440.

20. Jawed H, Anjum S, Awan SI, Simjee SU: Anti-arthritic effect of GN1, a novel synthetic analog of glucosamine, in the collagen-induced arthritis model in rats. Inflamm Res 2011, 60:1113-1120.

21. Hua J, Suguro S, Hirano S, Sakamoto K, Nagaoka I: Preventive actions of a high dose of glucosamine on adjuvant arthritis in rats. Inflamm Res 2005, 54:127-132

22. Dodge GR, Jimenez SA: Glucosamine sulfate modulates the levels of aggrecan and matrix metalloproteinase- 3 synthesized by cultured human osteoarthritis articular chondrocytes. Osteoarthr Cartil 2003, 11:424-432.

23. Byron CR, Stewart MC, Stewart AA, Pondenis HC: Effects of clinically relevant concentrations of glucosamine on equine chondrocytes and synoviocytes in vitro. Am J Vet Res 2008, 69:1129-1134.

24. Mobasheri A, Bondy CA, Moley K, Mendes AF, Rosa SC, Richardson SM, Hoyland JA, Barrett-Jolley R, Shakibaei M: Facilitative glucose transporters in articular chondrocytes. Expression, distribution and functional regulation of GLUT isoforms by hypoxia, hypoxia mimetics, growth factors and pro-inflammatory cytokines. Adv Anat Embryol Cell Biol 2008, 200(1):1-84. p following vi.

25. Mobasheri A, Richardson S, Mobasheri R, Shakibaei M, Hoyland JA: Hypoxia inducible factor-1 and facilitative glucose transporters GLUT1 and GLUT3: putative molecular components of the oxygen and glucose sensing apparatus in articular chondrocytes. Histol Histopathol 2005, 20:1327-1338

26. Mobasheri A: Role of chondrocyte death and hypocellularity in ageing human articular cartilage and the pathogenesis of osteoarthritis. Med Hypotheses 2002, 58:193-197.

27. Rosa SC, Goncalves J, Judas F, Mobasheri A, Lopes C, Mendes AF: Impaired glucose transporter-1 degradation and increased glucose transport and oxidative stress in response to high glucose in chondrocytes from osteoarthritic versus normal human cartilage. Arthritis Res Ther 2009, 11:R80.

28. Uitterlinden EJ, Jahr H, Koevoet JL, Bierma-Zeinstra SM, Verhaar JA, Weinans $H$, van Osch GJ: Glucosamine reduces anabolic as well as catabolic processes in bovine chondrocytes cultured in alginate. Osteoarthr Cartil 2007, 15:1267-1274.

29. Virkamaki A, Yki-Jarvinen H: Allosteric regulation of glycogen synthase and hexokinase by glucosamine-6-phosphate during glucosamine-induced insulin resistance in skeletal muscle and heart. Diabetes 1999, 48:1101-1107.

30. Tesoriere G, Vento R, Calvaruso G: Inhibitory effect of D-glucosamine on glycolysis in bovine retina. Biochim Biophys Acta 1975, 385:58-67.

31. Hinderlich S, Berger M, Schwarzkopf M, Effertz K, Reutter W: Molecular cloning and characterization of murine and human $\mathrm{N}$-acetylglucosamine kinase. Eur J Biochem 2000, 267:3301-3308.

32. Block JA, Oegema TR, Sandy JD, Plaas A: The effects of oral glucosamine on joint health: is a change in research approach needed? Osteoarthr Cartil 2010, 18:5-11.

33. Hubert C, Houari S, Lecomte F, Houbart V, De Bleye C, Fillet M, Piel G, Rozet E, Hubert P: Development and validation of a sensitive solid phase extraction/hydrophilic interaction liquid chromatography/mass spectrometry method for the accurate determination of glucosamine in dog plasma. J Chromatogr A 2010, 1217:3275-3281.

34. Roda A, Sabatini L, Barbieri A, Guardigli M, Locatelli M, Violante FS, Rovati LC, Persiani S: Development and validation of a sensitive HPLC-ESI-MS/ MS method for the direct determination of glucosamine in human plasma. J Chromatogr B Analyt Technol Biomed Life Sci 2006, 844:119-126.

35. Persiani S, Roda E, Rovati LC, Locatelli M, Giacovelli G, Roda A: Glucosamine oral bioavailability and plasma pharmacokinetics after increasing doses of crystalline glucosamine sulfate in man. Osteoarthr Cartil 2005, 13:1041-1049.

36. Biggee BA, Blinn CM, Nuite M, McAlindon TE, Silbert JE: Changes in serum levels of glucosamine and sulphate after ingestion of glucosamine sulphate with and without simultaneous ingestion of glucose. Ann Rheum Dis 2007, 66:1403-1404.

37. Zhong S, Zhong D, Chen X: Improved and simplified liquid chromatography/electrospray ionization mass spectrometry method for the analysis of underivatized glucosamine in human plasma. $J$ Chromatogr B Analyt Technol Biomed Life Sci 2007, 854:291-298.

38. Laverty S, Sandy JD, Celeste C, Vachon P, Marier JF, Plaas AH: Synovial fluid levels and serum pharmacokinetics in a large animal model following treatment with oral glucosamine at clinically relevant doses. Arthritis Rheum 2005, 52:181-191

39. Persiani S, Rotini R, Trisolino G, Rovati LC, Locatelli M, Paganini D, Antonioli $D$, Roda A: Synovial and plasma glucosamine concentrations in osteoarthritic patients following oral crystalline glucosamine sulphate at therapeutic dose. Osteoarthr Cartil 2007, 15:764-772.

40. Pastorini E, Rotini R, Guardigli M, Vecchiotti S, Persiani S, Trisolino G, Antonioli D, Rovati LC, Roda A: Development and validation of a HPLC-ES -MS/MS method for the determination of glucosamine in human synovial fluid. J Pharm Biomed Anal 2009, 50:1009-1014.

41. Jackson CG, Plaas AH, Sandy JD, Hua C, Kim-Rolands S, Barnhill JG, Harris CL, Clegg DO: The human pharmacokinetics of oral ingestion of glucosamine and chondroitin sulfate taken separately or in combination. Osteoarthr Cartil 2010, 18:297-302.

42. Largo R, Martinez-Calatrava MJ, Sanchez-Pernaute O, Marcos ME, MorenoRubio J, Aparicio C, Egido J, Herrero-Beaumont G: Effect of a high dose of glucosamine on systemic and tissue inflammation in an experimental model of atherosclerosis aggravated by chronic arthritis. Am J Physiol Heart Circ Physiol 2009, 297:H268-276.

43. Miller KL, Clegg DO: Glucosamine and chondroitin sulfate. Rheum Dis Clin North Am 2011, 37:103-118.

44. Largo R, Alvarez-Soria MA, Diez-Ortego I, Calvo E, Sanchez-Pernaute O, Egido J, Herrero-Beaumont G: Glucosamine inhibits IL-1beta-induced NFkappaB activation in human osteoarthritic chondrocytes. Osteoarthr Cartil 2003, 11:290-298.

45. Bruyere O, Pavelka K, Rovati LC, Deroisy R, Olejarova M, Gatterova J, Giacovelli G, Reginster JY: Glucosamine sulfate reduces osteoarthritis progression in postmenopausal women with knee osteoarthritis: evidence from two 3-year studies. Menopause 2004, 11:138-143.

46. Bruyere O, Honore A, Ethgen O, Rovati LC, Giacovelli G, Henrotin YE, Seidel $L$, Reginster JY: Correlation between radiographic severity of knee osteoarthritis and future disease progression. Results from a 3-year prospective, placebo-controlled study evaluating the effect of glucosamine sulfate. Osteoarthr Cartil 2003, 11:1-5

47. Bruyere O, Pavelka K, Rovati LC, Gatterova J, Giacovelli G, Olejarova M, Deroisy $R$, Reginster JY: Total joint replacement after glucosamine sulphate treatment in knee osteoarthritis: results of a mean 8-year observation of patients from two previous 3-year, randomised, placebocontrolled trials. Osteoarthr Cartil 2008, 16:254-260.

48. Wandel S, Juni P, Tendal B, Nuesch E, Villiger PM, Welton NJ, Reichenbach S, Trelle S: Effects of glucosamine, chondroitin, or placebo in patients with osteoarthritis of hip or knee: network meta-analysis. BMJ 2010, 341:c4675.

49. EFSA Panel on Dietetic Products NaA: Scientific Opinion on the substantiation of a health claim related to glucosamine and maintenance of joints pursuant to Article 13(5) of Regulation (EC) No 1924/2006. EFSA Journal 2011, 9:2476.

50. Ostojic SM, Arsic M, Prodanovic S, Vukovic J, Zlatanovic M: Glucosamine administration in athletes: effects on recovery of acute knee injury. Res Sports Med 2007, 15:113-124

51. Yoshimura M, Sakamoto K, Tsuruta A, Yamamoto T, Ishida K, Yamaguchi H, Nagaoka I: Evaluation of the effect of glucosamine administration on 
biomarkers for cartilage and bone metabolism in soccer players. Int J Mol Med 2009, 24:487-494.

52. Chen HC, Shah S, Stabler TV, Li YJ, Kraus VB: Biomarkers associated with clinical phenotypes of hand osteoarthritis in a large multigenerational family: the CARRIAGE family study. Osteoarthr Cartil 2008, 16:1054-1059.

53. Sowers M, Zobel D, Weissfeld L, Hawthorne VM, Carman W: Progression of osteoarthritis of the hand and metacarpal bone loss. A twenty-year followup of incident cases. Arthritis Rheum 1991, 34:36-42.

54. Goldring MB, Otero M: Inflammation in osteoarthritis. Curr Opin Rheumatol 2011, 23:471-478.

55. Moreau M, Lussier B, Doucet M, Vincent G, Martel-Pelletier J, Pelletier JP: Efficacy of Licofelone in dogs with clinical osteoarthritis. Vet Rec 2007 160(17):584-588

56. Pelletier JP, Martel-Pelletier J: In vivo protective effects of prophylactic treatment with tiaprofenic acid or intraarticular corticosteroids on osteoarthritic lesions in the experimental dog model. J Rheumatol Supp/ 1991, 27:127-130.

57. Raynauld JP, Martel-Pelletier J, Berthiaume MJ, Abram F, Choquette D, Haraoui B, Beary JF, Cline GA, Meyer JM, Pelletier JP: Correlation between bone lesion changes and cartilage volume loss in patients with osteoarthritis of the knee as assessed by quantitative magnetic resonance imaging over a 24-month period. Ann Rheum Dis 2008, 67:683-688.

58. Imagawa K, de Andres MC, Hashimoto K, Pitt D, Itoi E, Goldring MB, Roach $\mathrm{HI}$, Oreffo RO: The epigenetic effect of glucosamine and a nuclear factorkappa B (NF-kB) inhibitor on primary human chondrocytes-implications for osteoarthritis. Biochem Biophys Res Commun 2011, 405:362-367.

59. Chiusaroli R, Piepoli T, Zanelli T, Ballanti P, Lanza M, Rovati LC, Caselli G: Experimental pharmacology of glucosamine sulfate. Int J Rheumatol 2011, 2011:939265.

60. Baron AD, Zhu JS, Zhu JH, Weldon H, Maianu L, Garvey WT: Glucosamine induces insulin resistance in vivo by affecting GLUT 4 translocation in skeletal muscle. Implications for glucose toxicity. J Clin Invest 1995, 96:2792-2801.

61. Simon RR, Marks V, Leeds AR, Anderson JW: A comprehensive review of oral glucosamine use and effects on glucose metabolism in normal and diabetic individuals. Diabetes Metab Res Rev 2011, 27:14-27.

62. Puenpatom RA, Victor TW: Increased prevalence of metabolic syndrome in individuals with osteoarthritis: an analysis of NHANES III data. Postgrad Med 2009, 121:9-20.

63. Giaccari A, Morviducci L, Zorretta D, Sbraccia P, Leonetti F, Caiola S, Buongiorno A, Bonadonna RC, Tamburrano G: In vivo effects of glucosamine on insulin secretion and insulin sensitivity in the rat: possible relevance to the maladaptive responses to chronic hyperglycaemia. Diabetologia 1995, 38:518-524.

64. Scroggie DA, Albright A, Harris MD: The effect of glucosamine-chondroitin supplementation on glycosylated hemoglobin levels in patients with type 2 diabetes mellitus: a placebo-controlled, double-blinded, randomized clinical trial. Arch Intern Med 2003, 163:1587-1590.

65. Muniyappa R, Karne RJ, Hall G, Crandon SK, Bronstein JA, Ver MR, Hortin GL, Quon MJ: Oral glucosamine for 6 weeks at standard doses does not cause or worsen insulin resistance or endothelial dysfunction in lean or obese subjects. Diabetes 2006, 55:3142-3150.

66. Pham T, Cornea A, Blick KE, Jenkins A, Scofield RH: Oral glucosamine in doses used to treat osteoarthritis worsens insulin resistance. Am J Med Sci 2007, 333:333-339.

67. Biggee BA, Blinn CM, Nuite M, Silbert JE, McAlindon TE: Effects of oral glucosamine sulphate on serum glucose and insulin during an oral glucose tolerance test of subjects with osteoarthritis. Ann Rheum Dis 2007, 66:260-262.

68. Dostrovsky NR, Towheed TE, Hudson RW, Anastassiades TP: The effect of glucosamine on glucose metabolism in humans: a systematic review of the literature. Osteoarthr Cartil 2011, 19:375-380.

69. Monauni T, Zenti MG, Cretti A, Daniels MC, Targher G, Caruso B, Caputo M, McClain D, Del Prato S, Giaccari A, Muggeo M, Bonora E, Bonadonna RC: Effects of glucosamine infusion on insulin secretion and insulin action in humans. Diabetes 2000, 49:926-935.

70. Pouwels MJ, Jacobs JR, Span PN, Lutterman JA, Smits P, Tack CJ: Short-term glucosamine infusion does not affect insulin sensitivity in humans. J Clin Endocrinol Metab 2001, 86:2099-2103.
71. Tannis AJ, Barban J, Conquer JA: Effect of glucosamine supplementation on fasting and non-fasting plasma glucose and serum insulin concentrations in healthy individuals. Osteoarthr Cartil 2004, 12:506-511.

72. Towheed TE, Maxwell L, Anastassiades TP, Shea B, Houpt J, Robinson V, Hochberg MC, Wells G: Glucosamine therapy for treating osteoarthritis. Cochrane Database Syst Rev 2001(1):CD002946. Review. Update in: Cochrane Database Syst Rev. 2005;(2):CD002946. PMID:11279782.

73. Towheed TE, Maxwell L, Anastassiades TP, Shea B, Houpt J, Robinson V, Hochberg MC, Wells G: Glucosamine therapy for treating osteoarthritis. Cochrane Database Syst Rev 2005(2):CD002946. Review. PMID: 15846645.

74. Marshall S, Yamasaki K, Okuyama R: Glucosamine induces rapid desensitization of glucose transport in isolated adipocytes by increasing GlcN-6-P levels. Biochem Biophys Res Commun 2005, 329:1155-1161.

75. Gouze JN, Bianchi A, Becuwe P, Dauca M, Netter P, Magdalou J, Terlain B, Bordji K: Glucosamine modulates IL-1-induced activation of rat chondrocytes at a receptor level, and by inhibiting the NF-kappa B pathway. FEBS Lett 2002, 510:166-170.

76. Sandy JD, Gamett D, Thompson V, Verscharen C: Chondrocyte-mediated catabolism of aggrecan: aggrecanase-dependent cleavage induced by interleukin-1 or retinoic acid can be inhibited by glucosamine. Biochem J 1998, 335(Pt 1):59-66.

77. Piperno M, Reboul P, Hellio Le Graverand MP, Peschard MJ, Annefeld M, Richard M, Vignon E: Glucosamine sulfate modulates dysregulated activities of human osteoarthritic chondrocytes in vitro. Osteoarthr Cartil 2000, 8:207-212

78. Gouze JN, Gouze E, Popp MP, Bush ML, Dacanay EA, Kay JD, Levings PP, Patel KR, Saran JP, Watson RS, Ghivizzani SC: Exogenous glucosamine globally protects chondrocytes from the arthritogenic effects of IL-1beta. Arthritis Res Ther 2006, 8:R173.

79. Petersen SG, Beyer N, Hansen M, Holm L, Aagaard P, Mackey AL, Kjaer M: Nonsteroidal anti-inflammatory drug or glucosamine reduced pain and improved muscle strength with resistance training in a randomized controlled trial of knee osteoarthritis patients. Arch Phys Med Rehabil 2011, 92:1185-1193.

80. Mobasheri A, Henrotin Y: Biomarkers of osteoarthritis: a review of recent research progress on soluble biochemical markers, published patents and areas for future development. Recent Patents on Biomarkers 2011, $1: 25-43$

81. Henrotin Y, Deberg M, Mathy-Hartert M, Deby-Dupont G: Biochemical biomarkers of oxidative collagen damage. Adv Clin Chem 2009, 49:31-55

82. Anandacoomarasamy A, Leibman S, Smith G, Caterson I, Giuffre B, Fransen M, Sambrook PN, March L: Weight loss in obese people has structuremodifying effects on medial but not on lateral knee articular cartilage. Ann Rheum Dis 2012, 71:26-32

83. Kraus VB, McDaniel G, Worrell TW, Feng S, Vail TP, Varju G, Coleman RE: Association of bone scintigraphic abnormalities with knee malalignment and pain. Ann Rheum Dis 2009, 68:1673-1679.

84. Aghazadeh-Habashi A, Jamali F: The glucosamine controversy; a pharmacokinetic issue. J Pharm Pharm Sci 2011, 14:264-273.

\section{doi:10.1186/1756-0500-6-115}

Cite this article as: Henrotin et al:: Physiological effects of oral glucosamine on joint health: current status and consensus on future research priorities. BMC Research Notes 2013 6:115.

\section{Submit your next manuscript to BioMed Central and take full advantage of:}

- Convenient online submission

- Thorough peer review

- No space constraints or color figure charges

- Immediate publication on acceptance

- Inclusion in PubMed, CAS, Scopus and Google Scholar

- Research which is freely available for redistribution 